\title{
Uses and advantages of CRISPR/Cas genetic edition in yeasts
}

\author{
Amado Daniela ${ }^{1}$ Velez Norida ${ }^{1}$, Ceballos-Garzón Andres ${ }^{1}$, Juan Monrroy¹, Parra-Giraldo Claudia ${ }^{1}$
}

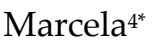

${ }^{1}$ Unidad de Investigación en Proteómica y Micosis Humanas, Grupo de Investigación en Enfermedades Infecciosas, Dpto. de Microbiología, Facultad de Ciencias, Pontificia Universidad Javeriana; Bogotá, Colombia.

\section{AD: d.amado@javeriana.edu.co}

VN: velez.norida@javeriana.edu.co

CGA: c-ceballos@javeriana.edu.co

JM: j_monroy@javeriana.edu.co

4*PGCM:claudia.parra@javeriana.edu.co

$4^{*}$ corresponding author: Claudia Marcela Parra Giraldo, Phone: 3006079718

\begin{abstract}
This review summarizes the use of CRISPR system in yeasts, identifying advantages and disadvantages of its applications. 39 articles were evaluated including 12 articles that discussed the advantages of new CRISPR systems that improved the initial system, and another 27 were evaluated, among these: three were applications in Cryptococcus neoformans, four in candida sp., three in Schizosaccharomyces pombe, nine in Saccharomyces cerevisiae, four in Yarrowia lipolytica, and four in industrially important yeasts such as Pichia pastoris and Saccharomyces pastorianus. It was concluded that the CRISPR system is one of the most versatile genetic editing systems available nowadays. It can be applied in different organisms for several effects including gene knock-outs, performing point mutations, gene expression, or even applying multiple edition operations in several genes. However, we recognize that numerous studies lack a control group of the mutated strains, which leaves many questions unanswered. For instance, the extent and precision of this techniques, it also represents a risk to biosecurity standards. Therefore, this review shows the compilation of CRISPR system information, which could be used to generate different alternatives in the industry and clinical fields
\end{abstract}

Keywords: CRISPR System, Yeast, gene edition 


\section{Introduction}

In the past, invasive fungal infections were rare enough to be thought of as unimportant. However, yeasts are able to colonize different environments rich in carbon compounds [1]. The study of pathogenic fungi is of great importance due to the increase of clinical cases and the few available antifungals. Additionally, there are weaknesses in the spectrum, potency, and pharmacokinetics of many therapeutic compounds. Research on the study of these pathogens focuses on their pathogenicity, virulence, and differentiation of fungal cells from mammalian host cells, due to their great similarity [2]. Yeasts are also known for their industrial applications which include food production and drug development. The genus with greater industrial use is Saccharomyces, it is also a frequent model for biotechnological studies [3].

The CRISPR system (clustered regularly interspaced short palindromic repeats) was identified as a defense mechanism against exogenous DNA molecules that could affect and alter the cell's DNA, such as phage or plasmid infections and it's thought of as an adaptive immunity system in prokaryotes. [1]. Over the years, many studies were conducted to understand the function of the system in prokaryotes and the structures of the molecules involved in this system. It was not until 2012 that Jennifer Doudna a Canadian researcher and Emmanuelle Charpentier a French researcher reinvented the CRISPR system and repurposed it as a tool for genetic engineering [2]. This system has brought a revolution upon contemporary biology in the modification of genetic information.

The CRISPR-CAS system is divided into 2 classes; those of class 1 are those that need a large complex of proteins that carry out the action of DNA degradation through a sequence of guide RNA. Those of class 2 are systems that need a single effector endonuclease protein guided by an RNA to carry out the neutralization of the invasive genome. This is the case of the CRISPR-Cas9 and CRISPR-Cpf1 system [3]. Cas9 endonuclease is the most used in genetic editing for Eukaryotic microorganisms such as yeasts. The class 2 system is composed of an endonuclease (Cas9 or Cpf1), a short sequence of RNA and the sequence PAMs short DNA sequences (from 3 to $5 \mathrm{bp}$ ) called protospacer adjacent motifs [4]. The endonuclease has two lobes: a) recognition system (REC), which is divided into two domains REC1 and REC2, and b) nuclease activity system (NUC), it has the RuvC, HNH and PI domains recognized by the PAM sequence in the DNA sequence [5]. This endonuclease protein performs its activity using the RuvC and $\mathrm{HNH}$ domains three nucleotides upstream of the PAM sequence. The guide RNA (sgRNA) consists of a chimeric RNA that retains two fundamental characteristics: a 5 'sequence that determines the DNA target site by pairing Watson-Crick bases (crRNA) and a 3' duplex RNA structure that binds to Cas9 (tracrRNA) [6]. In this review, we describe the application of this system in two species of yeasts of clinical importance, Candida sp, and Cryptococcus neoformans, as well as industrially important species Saccharomyces cerevisiae, Yarrowia lipolytica, and Schizosaccharomyces pombe.

2. Study of editing of genomes by CRISPR-Cas in yeasts of industrial and clinical importance. 
Candida Sp is a genus of yeasts and is the most common cause of fungal infections worldwide; This opportunistic infection has a global incidence of around 700,000 cases per year [4]. Although the genus has around 20 human pathogenic species, the CRISPR-Cas9 system to date has been used in only three species of them: C. albicans, C. glabrata, and C. parapsilosis.

The CRISPR-Cas9 system was used to modify the genes ADE2, MET15 and SOK2 in C. glabrata, which were located in different chromosomes. The study compared with the application of a SAT1 cassette, a more stablished method of genetic editing. They demonstrated that the CRISPR system is three times more efficient compared to the alternative method of genome editing [5]. The efficiency of CRISPR-Cas9 was not homogenous between all target genes, it was higher for MET15 compared to ADE2 for example. In addition, it is known that efficiency varies in the same target gene depending on the sgRNA sequence used [6].

In other study, the technique was applied to generate knock-out mutations in two genes of C. glabrata which were thought to be associated with the infection process. After successfully performing the desired mutations with a CRISPR-Cas9 system, the mutants were evaluated in the animal infection model D. melanogaster. Their results show the mutants were less virulent than the WT strains, concluding that these two genes must participate in the infection process of C. glabrata in vivo.[7].

The development of modifications in the CRISPR-Cas9 system is frequent. These modifications depend to a great extent on the known genotypic characteristics of the microorganism. In $C$. parapsilosis an editing system was developed that consisted of a single step of transformation, expressing the CAS9 gene only when the plasmid was present. in addition, this modification allowed to easily eliminate the transformed strains. It is the first system applied in C. parapsilosis using a marker of resistance to nourseothricin in which they published the genes (URA3 and ADE2) by consecutive transformation with two plasmids expressing different sgRNAs to increase the efficiency of gene editing. This gene editing/deletion system could be easily used and could be applied to generate a large number of genetic knock-outs [8].

Cryptococcus neoformans: Cryptococcosis is an opportunistic fungal infection, acquired by the inhalation of fungal propagules present in the environment; is a potentially fatal infection that affects the lungs and the central nervous system (CNS) in immunosuppressed and immunocompetent individuals [9]. The infection is caused by two species, namely: 1) Cryptococcus neoformans var. grubii (serotype A), var. neoformans (serotype D) and a hybrid corresponding to serotype AD and 2) Cryptococcus gattii (serotypes B and C). These species present phenotypic, genotypic and epidemiological differences, as well as in their geographic distribution, in addition, it is a mycosis that presents tropism by the CNS causing meningitis, different virulence factors have been described, implicated in pathogenicity as the capsular size and the production of melanin [10]. Therefore, several investigations have focused on the establishment of the CRISPR-Cas9 system to carry out the selective elimination of virulence and pathogenicity genes in Cryptococcus neoformans.

This yeast has a low frequency of homologous recombination, especially for strains of serotype D 
120

121

122

123

124

125

126

127

128

129

130

131

132

133

134

135

136

137

138

139

140

141

142

143

144

145

146

147

148

149

150

151

152

153

154

155

156

157

158

[11], which has hindered molecular genetic studies in the past. In 2016 Arras S., and collaborators evaluated the use of CRISPR Class 2 for the study of pathogenicity in Cryptococcus neoformans. Initially, they expressed a derivative of Streptococcus pyogenes nuclease Cas9 in C. neoformans and showed that it has no effect on growth, in addition, they evaluated the production of virulence factors in a murine model. They tested CAS9 in combination with multiple self-cleaving guide RNAs targeting the $A D E 2$ gene encoding phosphoribosilaminoamidazole carboxylase. This revealed that CRISPR functionality in C. neoformans depends on the CAS9 construct being stably integrated into the genome, whereas the transient expression of the guide RNA is enough to increase the rates of homologous recombination. This highlights the versatility of this genetic system. Furthermore, the presence of CRISPR nuclease does not influence virulence in a murine model, they successfully demonstrated that this system is compatible with pathogenicity studies in C. neoformans [12].

When the CRISPR-Cas9 system persists in the host cells, cytotoxicity effects may occur, which could block the performed genetic manipulation. This system is a powerful method to perform directed mutagenesis in organisms that present low recombination frequencies and for functional genomic studies. Wang Y., and collaborators reported a method to spontaneously eliminate the CRISPR-Cas9 system without affecting its robust editing function. They expressed the unique guiding RNA under the driver of an endogenous U6 promoter and the Cas9 endonuclease optimized in human codon with an ACT1 promoter. This system efficiently generated gene alteration through homologydirected repair by electroporation in yeasts, spontaneous elimination of the system was demonstrated through a CRISPR-Cas9 expression cis arrangement, allowing the validation of genetic functions through subsequent complementation and has the potential to minimize the effects outside the target [13].

The biobalistic transformation in C. neoformans, is a tool used for editing the genome where the introduced DNA is inherited in a stable manner, the transformation efficiency and the homologous integration rate is low (approximately 1-10\%). The development of Transient CRISPR -Cas9 together with the electroporation system (TRACE) proved useful to increase the rate of transformation since it efficiently integrated new material into the genome due to double-strand breaks created in specific sites by the CRISPR-Cas9 system and the high transformation efficiency of electroporation. This system can effectively eliminate multiple genes in a single transformation, as well as insert DNAs into a designated genetic site without any homologous sequence, which opens many other applications [14]. CRISPR can be used as a tool for the interruption of genes of high efficiency in C. neoformans, it is a useful system to understand this organism and its pathogenicity.

\section{Yeasts of industrial importance in biotechnological processes}

Saccharomyces cerevisiae: It is the most used yeast in industrial processes, followed by S. bayanus and S. pastorianus. In addition, it is the best studied yeast both in its physiological and genetic characteristics, it was even the first Eukaryotic cell to be sequenced [15]. In the industry editing of its genome has been implemented in the past to optimize processes among other applications. Studies have been conducted using the CRISPR-Cas9 technology to reduce production of ethyl carbamate (EC), a potential carcinogen which is formed during the process of fermentation of ethanol in this 
yeast. It has also been used to perform a "knock out" of the CAR1 gene involved in the synthesis of the EC. Process, leaving no antibiotic marker genes or any residual sequence in the vicinity of the CAR1 gene. To inactivate the gene, they introduced a nonsense mutation in the start codon of glycine (Gln) transforming it into a stop codon (TAA) by homologous recombination and designed another experiment that performed the elimination of the traditional NHEJ, eliminating $1002 \mathrm{bp}$ of the gene. To achieve their goal they used two plasmids, one containing the CRISPR system and the other containing the sgRNA. They demonstrated that CRISPR-Cas9-mediated inactivation of the CAR1 gene led to a significant reduction in the specific activity of arginase, urea and CD. Furthermore, when comparing the phenotype of the mutants with the wild-type strains, they did not observe statistically significant differences in the growth of the yeast in the culture, nor in the percentage of glucose consumption and ethanol production, which shows that the mutation did not affect the industrial yield of the production, and on the contrary, it reduced the levels of CE by $60 \%$ in culture [16].

In recent years, new CRISPR designs have been generated in S. cerevisiae. Dashko $S$ and collaborators in 2018, implemented a new family of programmable endonucleases by CRISPR-Cas class II. Using the constitutively expressed endonuclease Cpf1 of Francisella novicida (Fn Cpf1), RNA cleavage of DNA guided by specific genomic loci can mediate. When evaluating this nuclease, they showed that it is not toxic to the yeast and does not interfere with the growth in the culture medium. They determined that the PAM sequence that recognizes this endonuclease is TTN and that Fn Cpf1 is able to perform a directed edition guided by a crRNA and in turn perform point mutations in the ADE2 gene by inserting a stop codon. Fn Cpf1 showed an efficiency of up to $100 \%$ for the repair of DNA recombination [17].

A novel method for the editing of the genome by CRISPR-Cas9 in S. cerevisiae, consists of introducing a cut-off site in a specific genomic location, followed by the integration of a sequence edited in the same location in a way without scars. They published sequences of the promoter GAL1 and GAL80, they managed to over-express both genes in the yeast, and they showed that the production of agrosa was proportional to the fluorescence emitted by the marker, to achieve this method they had to insert the PAM sequence in the region in a synthetic way. close to the promoter to achieve that the CRISPR system will identify the site in which it should be inserted to stimulate the promoter and overextend the gene [18]. Protocols have also been developed that produce integrations without scars and without DNA markers using a 20 bp sgRNA immediately following the PAM (NGG) sequence [19].

The CRISPR-Cas9 multiplex system was used for the genome engineering of up to 5 different genomic loci in a single transformation step in the yeast, with this methodology. Jakočinas $\mathrm{T}$ et al; in 2015, managed to overexpress 41 times the mevalonate, an important intermediate metabolite in cholesterol biosynthesis in mutated strains, which makes this methodology a useful tool to increase the production of an industrial molecule[20]. In addition, libraries of high efficiency integration plasmids have been generated to be implemented in the CRISPR-Cas9 system. The DOE Joint BioEnergy Institute, Emeryville of California used a set of cloning-free tools that allows rapid and easy genetic modification of strains in S. cerevisiae, using different plasmids, which presented an 
efficiency above $95 \%$ in 23 genomic loci characterized. The toolkit described in this paper provides a rapid approach to examine multiple gene expression contexts simultaneously [21].

In Denmark they developed kit with EasyClone-MarkerFree vectors using the CRISPR / Cas9 system that facilitates the integration of linearized expression cassettes at defined genomic loci, expressing the (sgRNA) from a set of RNAG helper vectors. Using that set of genomic engineering vectors, simple inserts are obtained with $90-100 \%$ and triple with $60-70 \%$ focusing efficiency. The EasyCloneMarkerFree vector toolkit can be used to simultaneously enter one to three integration cassettes into the genome of S. cerevisiae, without the use of selection markers. Integration cassettes can be constructed for overexpression of one or two genes per integration site; In that study, they successfully integrated up to six genes in a single transformation with a targeting efficiency of 60 to $70 \%[22]$.

Using the CRISPR-Cas9 system, they integrated by homologous recombination (HR) the XYL1, XYL2 and XYL3 genes in the loci PHO13 and ALD6 (involved in the production of acetate), to achieve the overexpression of heterologous genes and the cancellation of endogenous genes simultaneously in strains of $S$. cerevisiae. For the construction of the mutated strain, they used the sgRNA directed to PHO13 and ALD6 sequentially to replace the genes; in the study they suggest that the sequential integration process can be shortened by a transformation with a plasmid carrying both sgRNA together using multiplex methods. All of the above was carried out in order to generate at an industrial level strains that have higher levels of fermentation and that do not represent a public health hazard, since they do not present resistance genes inserted in the mutation process [23].

S. cerevisiae, has been used in biotechnological processes, an example of this is the evaluation to the resistance to an antiparasitic against the malaria of the family spiroindolonas, the disadvantage with this medicine is that it becomes less active by mutations in an ATPase type P of the parasite. To verify this pattern in the parasite and that the mutations in the P-type ATPase enzyme confers resistance, they used the cellular model of S. cerevisiae, mutated (using CRISPR) the gene that encodes a P-type ATPase (ScPMA1 and ScYRR1) and exposed the cells mutated to spiroindolones (KAE609) to see if they acquired the resistance. These experiments confirmed that mutations in ScPMA1 and ScYRR1 cause a 2.5 fold increase in resistance to KAE609, however, ScYRR1 does not appear to be the main target of KAE609. On the other hand it was shown that the ScPMA1 gene mutation is the target molecule of a KAE609 and its mutation is directly related to the resistance [24].

CRISPR in S. cerevisiae, is a very useful system at an industrial level, it has been implemented for the editing of the genome with different approaches, the expression of genes that produce a harmful molecule have been inhibited, this technique was also used in the overexpression of genes and in bioengineering. S. cerevisiae, whose genome and functionality of its genes are well known, is a cellular model, very useful for the design of new CRISPR variant methodologies that allow multiplex mutation and open the possibility of generating more profitable strains for the industry in short time and in a very simple way. 
concentrations of lipids in its interior and its ability to secrete proteins to produce valuable biochemical products at an industrial level [25]. The work in this yeast has focused on its physiological, metabolic and genomic characteristics, specifically in the secretion of proteins, for the use of hydrophobic substrates and the biogenesis of the peroxisome, in addition, of the study of molecules involved in dimorphism, in understanding the Mitochondrial complexity, the biogenesis of the lipid body and lipid homeostasis, advances in the study of molecular biology have been made by explaining the splicing of introns and the alternative splice, among others[26].

Being a microorganism completely sequenced and with great industrial advantages, studies have been carried out in which the CRISPR gene editing technique has been implemented to mutate specific genes that improve the production of certain compounds in this yeast. In China, they implemented the CRISPR system to edit the genome with a single plasmid (pCAS1yl or pCAS2yl) by homologous recombination (RH) and non-homologous recombination (NHEJ) for the TRP1 and PEX10 genes [27]. They demonstrated that the highest percentage of efficiency of the system occurred on day 4 of growth, they achieved a simultaneous double and triple gene editing with the plasmid pCAS1yl by NHEJ. The system with the plasmid pCASyl was successful in different strains of Y. Lipolytica, therefore, this system was more efficient than traditional methods of genome editing and facilitated synthetic biology, metabolic engineering and functional genomic studies.

Zhang J Lai and Col in 2018, designed a new CRISPR system called CRISPRi multiplex system for the repression of multiple genes in a single step, in Y. Lipolytica, using the Golden-brick assembly method that can assemble different parts without the Initial PCR procedure, which prevents the introduction of new errors in the PCR amplification process and only needs two restriction enzyme sites so that all parts can be assembled in one step [28]. For the design of the CRISPRi system four repressors were used: Cpf1 deactivated with DNase (dCpf1) from Francisella novicida, Cas9 deactivated (dCas9) from Streptococcus pyogenes and two fusion proteins (dCpf1-KRAB and dCas9-KRAB); In addition, ten gRNAs that were linked to different regions of the GFP gene (green fluorescent protein) were designed and the results indicated that there was no clear correlation between the efficiency of repression and the target sites, regardless of which repressor protein was used. In order to rapidly produce strong gene repression, a multiplex sgRNA strategy was developed in which a high repression efficiency of $85 \%$ (dCpf1) and $92 \%$ (dCas9) was achieved in a short time by making three different gRNAs towards the GFP gene simultaneously.

In this same study, they repressed plural genes vioA, vioB and saw simultaneously and the gene saw using the CRISPRi multiplex system in Y. lipolytica. To test the effectiveness of the system, they constructed a VioABE strain containing the protodeoxy-violaceinic acid route (PVA, a pigment derived from tryptophan and its content can be quantified by absorbance) using the dCpf1-Multi and dCas9-Multi vectors, with simple sgRNA and with sgRNA multiplex ( 3 sgRNA). The transformation was evaluated by PVA in which it was evidenced that when repressing only vioE, the absorbance was reduced to $60 \%$ and $40 \%$ with the protein dCpf1 and the protein dCas 9 respectively, the CRISPRi multiplex system was feasible to implement the repression of multiple genes. 
Several protocols have been generated for the genetic editing of Y. lipolytica. In 2016b Jassop et al., Created a new genetic tool Easy-Clone YALI, which allows the construction of genetically modified strains with high efficiency in a simplified way in Y. lipolytica through the CRISPR / Cas9 technology. Modulates gene expression with the integration of cassettes in intergenic sites IntC_2, IntC_3, IntD_1, IntE_1 and IntE_3, in addition, did not affect the growth of yeast [29].

In yeast Y. Lipolytica, the CRISPR-Cas9 system was used to efficiently perform genome alteration, in most articles it was used as LEU2 selection marker, and the PCR technique was used to confirm the alteration of the genome. ; The transformation protocols in the four articles were very similar to each other and are a very useful tool to evaluate the efficiency of these protocols in other yeast species.

Schizosaccharomyces pombe: It is a yeast highly studied at an industrial and clinical level together with S. cerevisiae, which is widely used at an industrial level for the fermentation of alcoholic beverages such as rum, tequila, and artisan beverages such as Cachaça in Brazil [31]. It is also a yeast used in the wine industry due to its ability to use malic acid and thus reduce the acidity of wine [32]. In addition, it is a fully sequenced microorganism which allows biotechnological studies.

Jacobs JZ y cola in 2018 at the State University of New Jersey developed a CRISPR-Cas9 system that allowed the genome editing in S. pombe. They published the ade6 gene, being mutated causes the accumulation of a red precursor in media with low adenine content to be able to identify the transformed cells, concluded that the CRISPR-Cas9 mutagenesis achieves an almost complete efficiency and eliminates the need for selectable markers. The only vector that expresses Cas 9 and sgRNA is marked with URA4, which allows the elimination of the plasmid by selection with 5fluoroorotic acid and allows the subsequent mutagenesis of additional targets. The built-in rrk1 / Hammerhead Ribozyme cassette, expressed in Pol II, is useful in other situations where RNA expression and defined arbitrary sequences, such as siRNA or lincRNA, is needed and represents an advantage over Pol III RNA systems. The methods and reagents presented here are useful for genomic research in S. pombe by allowing rapid and specific genome editing[33].

In 2018, they described the CRISPR-Cas9 system to rapidly introduce deletions in the DNA regions that serve as auxotrophic markers in S. pombe, these were: leu1-D0, his3-D0 and lys9-D0, ura4-D18. This system consists of a Cas9 gRNA expression vector and a pair of donor DNA plasmids for each deletion. In addition, they reorganized the essential components in the commonly used pREP plasmid series and assembled the corresponding auxotrophic marker gene in these plasmids[34].

The developed a cloning-free procedure that uses DNA repair in fission S. pombe cells to assemble two linear DNA fragments was performed in 2018 in Beijing, used a circular plasmid encoding Cas 9 and an amplified sgRNA insert by PCR, both fragments contain only a portion of the URA4 or bsdMX marker, so that only the properly assembled plasmid can confer prototrophy (ability to grow on minimal medium) of uracil or resistance to blasticidin in transformed yeast [35]. In this study they showed that CRISPR-Cas9 based on repair and cloning free allows rapid and efficient point mutation, endogenous $\mathrm{N}$-terminal labeling and elimination of the genomic sequence in fission yeast. 
313

314

315

316

317

318

319

320

321

322

323

324

325

326

327

328

329

330

331

332

333

334

335

336

337

338

339

340

341

342

343

344

345

346

347

348

349

350

351

In the yeast Schizosaccharomyces pombe in recent years have been developed multiple studies that design various protocols and different mutation alternatives through the CRISPR / Cas9 system that facilitate the mutation of the genome and improve its efficiency to develop genomic studies that explain the function of different genes and at the same time can be used for other cellular models.

\section{CRISPR-Cas system in atypical yeasts.}

At present there are described, approximately 900 yeast species, the investigations are focused on a limited number of these species, we wanted to collect some studies on yeasts that are rare as is the case of Pichia pastoris and Saccharomyces pastorianus, specifically in the genomic edition by CRISPRCas, we reflect an overview of the latest advances in genomic editing in these yeasts, their main applications and the main challenges.

Pichia pastoris: reclassified as Komagataella pastoris, widely used today in the biotechnological field for the production of heterologous proteins [36]. In Austria they demonstrated the integration of cassettes with donor DNA without markers in the wild type strain of $P$. pastoris, which allows new engineering strategies. They tested three variants of Cas9 (Sp Cas9, Pp Cas9, Hs Cas9) available, to be evaluated in the pastorku70 strain of $P$. pastoris, they wanted to determine if they would need a different level of expression. For the wild-type strain only Hs Cas9 was included and gave the Cas9/ gRNA expression plasmids a Geneticin resistance marker which proved to be a versatile tool for the recycling of markers. The CRSIPR-Cas9 tool can be applied to modify the existing production strains and also open the way for studies of complete genome modification without markers in P. pastoris. In addition, it can be implemented for autonomous replication sequences (ARS) in classic knockout cassettes to guarantee cassette maintenance, especially in cell cycles in the S / G2 phase where HR predominates and, therefore, favor their integration[37].

In 2016 the same Austrian research group mentioned above, systematically tested more than 90 constructs containing different DNA sequences optimized with Cas9 codons, several gRNA sequences and promoters of Pol III RNA and Pol II RNA (in combination with ribozymes) for the expression of the gRNAs with different promoters of Pol II RNA for the expression of Cas9 and gRNAs, in order to identify the system failures in $P$. pastoris. They managed to generate an optimized system for this model which allows alter genes, introduce deletions of multiplexed genes and test the targeted integration of homologous DNA cassettes [38].

Saccharomyces pastorianus: it is widely used for brewing and in recent years its interest has been related to certain glycolysis processes. In the Netherlands, a method based on the CRISPR-Cas9 genome editing was designed for the precise elimination of genes (SeATF1 and SeATF2) in S. pastorianus. The cas9 gene expressed from a mobile genetic element was combined in combination with a plasmidtransmitted gRNA expression cassette, the expression of a gRNA flanked with Hammerhead ribozymes and delta Delta viruses using the TDH3 promoter dependent on RNA polymerase II successfully led to the precise elimination of the four alleles of SeILV6 in the strain CBS1483, in addition, the expression of two gRNAs flanked by ribozymes separated by a 10 bp linker in a polycistronic matrix successfully led to the simultaneous elimination of SeATF1 and SeATF2 genes, located in two separated chromosomes[39]. The compilation of these studies of editing of the genome 
352

353

354

355

356

357

358

by CRISPR-Cas in washes, allowed to consolidate a general vision of the advances of this technology of showing versatility in editing and approaches, which has allowed the creation of a CRISPRi strain collection for more than $99 \%$ of the genes needed for fermentative or respiratory growth [40].

The aforementioned studies on the genome edition in yeasts of industrial, biotechnological or clinical interest are of great importance to understand the biology of fungal cells in fermentative processes in the industry, important pathogenic processes for clinical studies or pharmacokinetic processes. CRISP-Cas is a new methodology implemented in yeasts, there is still a lot to explore and learn.

\section{Development of various approaches for the use of the CRISPR / Cas system}

Since 2012, the CRISPR-Cas system has been used for gene editing and its use has been extended to different species. In recent years, methodologies have been designed that use different CRISPR systems depending on the modifications that are to be made in the genome, here we collect some of the genome editing modifications (Table 1).

Table 1 New CRISPR methodologies.

\begin{tabular}{|c|c|c|c|}
\hline Technology & Name & Functions & Reference \\
\hline Di-CRISPR & $\begin{array}{l}\text { Delta Integration } \\
\text { CRISPR/Cas }\end{array}$ & $\begin{array}{l}\text { Integration of biochemical pathways in one step, without } \\
\text { markers for Cas9, by selecting repeated delta sites. }\end{array}$ & {$[41]$} \\
\hline CasEMBLR & CasEMBLR & $\begin{array}{l}\text { Multi-genomic integration loci facilitated by Cas9 from in-vivo } \\
\text { assembled parts. }\end{array}$ & [42] \\
\hline CRISPR-AID & $\begin{array}{l}\text { CRISPRa, CRISPRi } \\
\text { and CRISPRd }\end{array}$ & $\begin{array}{l}\text { It allows to increase the activation of a gene to overexpress } \\
\text { metabolic compounds. Several metabolic engineering targets can } \\
\text { be used for optimization and the exploration of synergistic } \\
\text { interactions between transcriptional activation, transcriptional } \\
\text { interference and gene elimination can be used. }\end{array}$ & [43] \\
\hline CRISPR-Cpf1 & Cpf1 & $\begin{array}{l}\text { It allows to increase the activation of a gene to overexpress } \\
\text { metabolic compounds. Several metabolic engineering targets can } \\
\text { be used for optimization and the exploration of synergistic } \\
\text { interactions between transcriptional activation, transcriptional } \\
\text { interference and gene elimination can be used. }\end{array}$ & {$[44]$} \\
\hline CRISPR-PCS & $\begin{array}{l}\text { Division of } \\
\text { chromosomes } \\
\text { mediated for PCR }\end{array}$ & $\begin{array}{l}\text { Improves the efficiency of chromosome division almost } 200 \\
\text { times. Allowing to create new strains with specific traits for } \\
\text { industrial applications and investigate the function of the } \\
\text { genome. }\end{array}$ & {$[45]$} \\
\hline dCas9-VPR & $\begin{array}{l}\text { Cas9 with the } \\
\text { chimeric activation } \\
\text { domain VPR. }\end{array}$ & $\begin{array}{l}\text { It allows performing transcriptional repression or transcriptional } \\
\text { activation. }\end{array}$ & [46] \\
\hline TAR-CRISPR & $\begin{array}{l}\text { Recombination } \\
\text { associated with } \\
\text { transformation }\end{array}$ & $\begin{array}{l}\text { It allows to isolate any chromosomal region by homologous } \\
\text { recombination, between mammalian cells and yeasts such as } \\
\text { S. cerevisiae }\end{array}$ & [47] \\
\hline CRISPRm. & $\begin{array}{l}\text { CRISPR multiplex } \\
\text { system }\end{array}$ & Allows you to edit multiple loci simultaneously & [48] \\
\hline CRISPRa & Activator & Allows transcriptional activation & [49] \\
\hline mCRISTAR & CRISPR Cas9 y TAR & $\begin{array}{l}\text { It allows the multiplexed substitution of promoters in groups of } \\
\text { biosynthetic genes avoiding the regulatory elements of } \\
\text { transcription, inducing gene expression }\end{array}$ & [50] \\
\hline dCas9 & Cas9 deactivate & Allows gene repression in a high efficiency repression site & $\begin{array}{l}{[51]} \\
{[52]}\end{array}$ \\
\hline
\end{tabular}


From the modifications made to CRISPR and its implementation in the edition of the genome in yeasts, we found that the system is very dynamic, adapts to almost any experimental design and can be implemented for any genetic modification that is desired; each of these variants improves the efficiency of the system and facilitates the editing process.

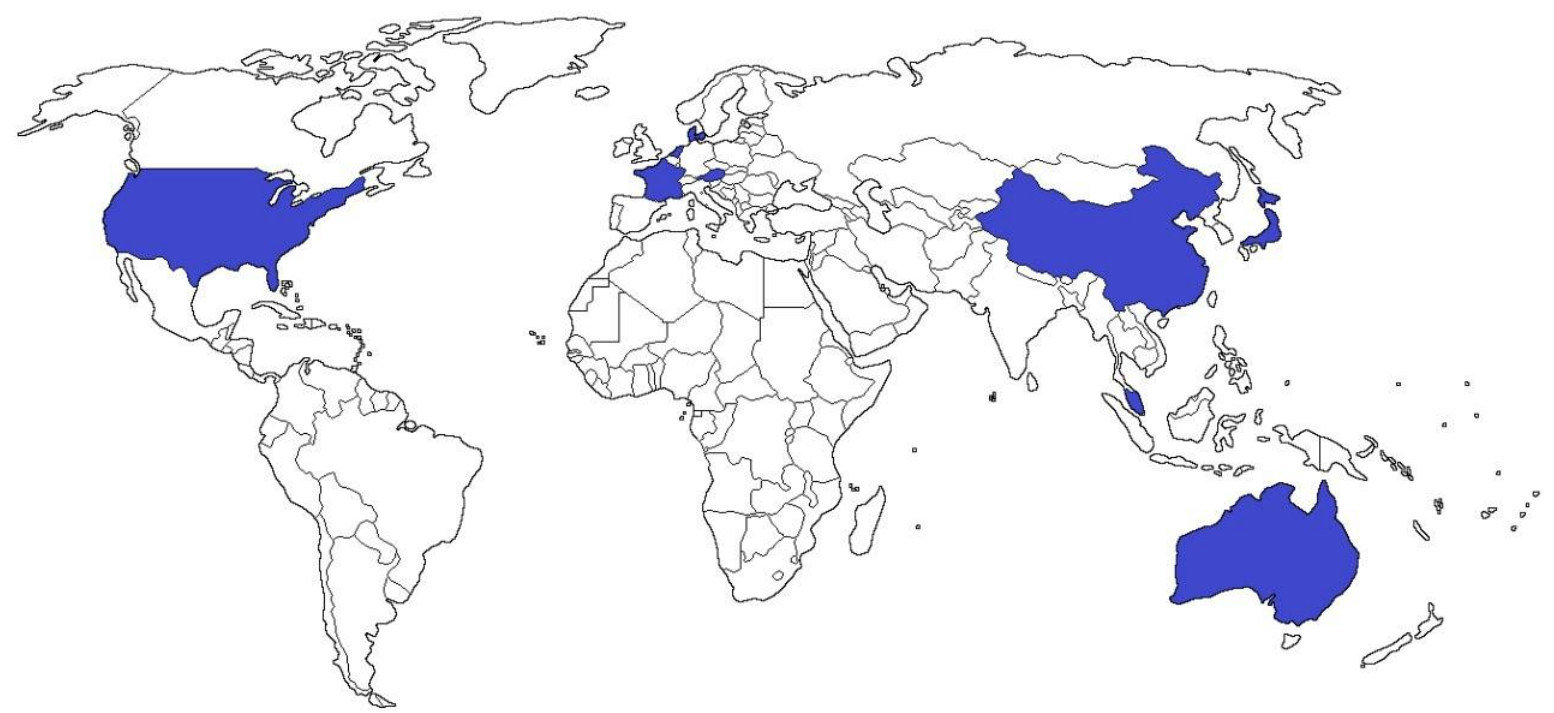

Figure 1. Mundial distribution with information about CRISPR-Cas system in yeast.

\section{Conclusion}

The CRISPR system is currently one of the most diverse genetic editing systems. It allows gene edition in different organisms as long as an efficient computer design of the sgRNA sequences is available. It will be implemented to achieve the objective of the study, be it to eliminate a gene, make point mutations, express a gene or do the aforementioned in several genes simultaneously. On the other hand, the new kits generated for this system have certain limitations. For example, the Easy-CloneMarkerFree kit designed in Denmark, where they obtained several integration cassettes simultaneously into the yeast genome.

One of the major concerns in the use of CRISPR-Cas is the management of modified strains, in most cases, the authors use control points in which they can elucidate the changes made, and in some cases, indicate when they were lost. However, in some investigations the management of these strains is not clear, although CRISPR / CAS is a technology that has provided many benefits in the genome edition thanks to its highly specificity and efficiency compared to the edition of TALENS and SINES. Its rapid evolution leaves little time for ethical and biosecurity controls, since organisms published by this technology could alter ecosystems if they are not properly managed.

As underlined in these articles, CRISPR is a widely used tool for better industrial processes, either to increase production or to eliminate some toxic compound that is generated during the production process of some molecule or industrial product in the yeasts are needed to synthesize or ferment them as it is in the case of alcoholic beverages, in addition, in the clinical area this tool has facilitated the study of several genes involved in signaling pathways that confer the yeast, either resistance treatment pharmacology, or confers pathogenicity on the host. Allowing thus generate alternatives for the industry and in clinic to problems that affect the human being.

\section{Supplementary Materials:}


Methodology

Search strategy: the PubMed, Lilacs and google academic databases were consulted. The inclusion criteria were all articles that included the CRISPR /Cas system in yeast, date restriction was not used, articles were consulted only in English and Spanish, searches were made through MeSH terms and Boolean operators, the terms of exclusion were other microorganisms different from yeast and other genome editing methodologies.

Selection of the articles: it was carried out according to the PRISMA methodology, it consists of a minimum set of elements, based on evidence, to help to present reports of systematic reviews, and it is useful for the critical evaluation of articles. The articles were evaluated and verified independently by four evaluators taking into account the title, summary, and reading of articles (See graphic prism Annex 1).

Analysis of articles: The articles were classified according to the type of study, yeast species and CRISPR / CAS technology type. The data was tabulated in the Excel program.

Author Contributions: formal analysis, VN, AD; data curation, MJ; writing-original draft preparation, VN, AD, and CGA. PGCM; supervision, PGCM.

Funding: This research received no external funding

\section{Acknowledgments:}

Conflicts of Interest: The authors declare that no competing interests existed.

\section{References}

1. Zhang H-Z, Gan L-L, Wang H, Zhou C-H. New Progress in Azole Compounds as Antimicrobial Agents. Mini Rev Med Chem. 2017;17:2.

2. Roque L, Molpeceres J, Reis C, Rijo P, Reis CP. Past, Recent Progresses and Future Perspectives of Nanotechnology Applied to Antifungal Agents. Curr Drug Metab. 2017;

3. Ghaemmaghami S, Huh W-K, Bower K, Howson RW, Belle A, Dephoure N, et al. Global analysis of protein expression in yeast. Nature. 2003 Oct;425:6959. doi:10.1038/nature02046

4. Bongomin F, Gago S, Oladele RO, Denning DW. Global and Multi-National Prevalence of Fungal Diseases-Estimate Precision. J fungi (Basel, Switzerland). 2017 Oct;3:4. doi:10.3390/jof3040057

5. Cen Y, Timmermans B, Souffriau B, Thevelein JM, Van Dijck P. Comparison of genome engineering using the CRISPR-Cas9 system in C. glabrata wild-type and lig4 strains. Fungal Genet Biol. 2017;107. doi:10.1016/j.fgb.2017.08.004

6. Min K, Ichikawa Y, Woolford CA, Mitchell AP. Candida albicans Gene Deletion with a Transient CRISPR-Cas9 System. 2016; doi:10.1126/sciadv.1500248

7. Enkler L, Richer D, Marchand AL, Ferrandon D, Jossinet F. Genome engineering in the yeast pathogen 
428

429

430

431

432

433

434

435

436

437

438

439

440

441

442

443

444

445

446

447

448

449

450

451

452

453

454

455

456

457

458
Candida glabrata using the CRISPR-Cas9 system. Sci Rep. 2016;6. doi:10.1038/srep35766

8. Lombardi L, Turner SA, Zhao F, Butler G. Gene editing in clinical isolates of Candida parapsilosis using CRISPR/Cas9. Sci Rep. 2017; doi:10.1038/s41598-017-08500-1

9. Mesquita M, Teixeira FM, Schalcher TR, Thielli M, Magalhães F, Valério ES, et al. Cryptococcosis , A Risk for Immunocompromised and Immunocompetent Individuals. 2013;

10. Cogliati M, Fett JP, Liao D, Taylor-Robinson A. Global Molecular Epidemiology of Cryptococcus neoformans and Cryptococcus gattii: An Atlas of the Molecular Types. Artic ID. 2013;2013. doi:10.1155/2013/675213

11. Davidson RC, Cruz MC, Sia RAL, Allen B, Alspaugh JA, Heitman J. Gene Disruption by Biolistic Transformation in Serotype D Strains of Cryptococcus neoformans. Fungal Genet Biol. 2000 Feb;29:1. doi:10.1006/FGBI.1999.1180

12. Arras SDM, Chua SMH, Wizrah MSI, Faint JA, Yap AS, Fraser JA. Targeted Genome Editing via CRISPR in the Pathogen Cryptococcus neoformans. 2016; doi:10.1371/journal.pone.0164322

13. Wang Y, Wei D, Zhu X, Pan J, Zhang P, Huo L, et al. A “suicide" CRISPR-Cas9 system to promote gene deletion and restoration by electroporation in Cryptococcus neoformans. Nat Publ Gr. 2016; doi:10.1038/srep31145

14. Fan Y, Lin X. Multiple Applications of a Transient CRISPR-Cas9 Coupled with Electroporation (TRACE) System in the Cryptococcus neoformans 2 Species Complex 3 Running title: No more biolistic transformation in Cryptococcus. 2018;706. doi:10.1534/genetics.117.300656

15. A. Goffeau, B. G. Barrell, H. Bussey, R. W. Davis, B. Dujon, H. Feldmann, F. Galibert, J. D. Hoheisel, C. Jacq MJ, White O, Clayton R, et al. Life with 6000 Genes. Science (80- ). 1995 Jul;269:5223. doi:10.1126/science.7542800

16. Chin YW, Kang WK, Jang HW, Turner TL, Kim HJ. CAR1 deletion by CRISPR/Cas9 reduces formation of ethyl carbamate from ethanol fermentation by Saccharomyces cerevisiae. J Ind Microbiol Biotechnol. 2016;43:11. doi:10.1007/s10295-016-1831-x

17. Dashko S, den Ridder M, Wijsman M, van der Oost J, Daran J-M, Daran-Lapujade P. FnCpf1: a novel and efficient genome editing tool for Saccharomyces cerevisiae. Nucleic Acids Res. 2017;45:2. doi:10.1093/nar/gkx1007

18. Elison GL, Song R, Acar M. A Precise Genome Editing Method Reveals Insights into the Activity of Eukaryotic Promoters. Cell Rep. 2017;18:1. doi:10.1016/j.celrep.2016.12.014

19. Ryan OW, Poddar S, Cate JHD. Crispr-cas9 genome engineering in Saccharomyces cerevisiae cells. Cold Spring Harb Protoc. 2016;2016:6. doi:10.1101/pdb.prot086827 
460

461

462

463

464

465

466

467

468

469

470

471

472

473

474

475

476

477

478

479

480

481

482

483

484

485

486

487

488

489

490

491

20. Jakočinas T, Bonde I, Herrgård M, Harrison SJ, Kristensen M, Pedersen LE, et al. Multiplex metabolic pathway engineering using CRISPR/Cas9 in Saccharomyces cerevisiae. Metab Eng. 2015;28. doi:10.1016/j.ymben.2015.01.008

21. Reider Apel A, d'Espaux L, Wehrs M, Sachs D, Li RA, Tong GJ, et al. A Cas9-based toolkit to program gene expression in Saccharomyces cerevisiae. Nucleic Acids Res. 2017 Jan;45:1.

doi:10.1093/nar/gkw1023

22. EasyClone-MarkerFree: A vector toolkit for marker-less integration of genes into Saccharomyces cerevisiae via CRISPR-Cas9. 2016; doi:10.1002/biot.201600147

23. Tsai CS, Kong II, Lesmana A, Million G, Zhang GC, Kim SR, et al. Rapid and marker-free refactoring of xylose-fermenting yeast strains with Cas9/CRISPR. Biotechnol Bioeng. 2015;112:11.

doi:10.1002/bit.25632

24. Goldgof GM, Durrant JD, Ottilie S, Vigil E, Allen KE, Gunawan F, et al. Comparative chemical genomics reveal that the spiroindolone antimalarial KAE609 (Cipargamin) is a P-type ATPase inhibitor OPEN. 2016; doi:10.1038/srep27806

25. Coelho MAZ, Amaral PFF, Belo I. Yarrowia lipolytica: an industrial workhorse.

26. Nicaud J-M. Yarrowia lipolytica. Yeast. 2012 Oct;29:10. doi:10.1002/yea.2921

27. Gao S, Tong Y, Wen Z, Zhu L, Ge M, Chen D, et al. Multiplex gene editing of the Yarrowia lipolytica genome using the CRISPR-Cas9 system. J Ind Microbiol Biotechnol. 2016;43:8. doi:10.1007/s10295-016$1789-8$

28. Zhang J lai, Peng YZ, Liu D, Liu H, Cao YX, Li BZ, et al. Gene repression via multiplex gRNA strategy in Y. lipolytica. Microb Cell Fact. 2018;17:1. doi:10.1186/s12934-018-0909-8

29. Dam MI, Dahlin J. EasyCloneYALI : CRISPR / Cas9-based synthetic toolbox for engineering of the yeast b Present a Present address : ALK-Abelló Nordic A / S , Bøge Allé 6-8 , DK-2970 Hørsholm ,.

30. Schwartz C, Shabbir-Hussain M, Frogue K, Blenner M, Wheeldon I. Standardized Markerless Gene Integration for Pathway Engineering in Yarrowia lipolytica. ACS Synth Biol. 2017;6:3. doi:10.1021/acssynbio.6b00285

31. Gomes FCO, Pataro C, Guerra JB, Neves MJ, Corrêa SR, Moreira ESA, et al. Physiological diversity and trehalose accumulation in Schizosaccharomyces pombe strains isolated from spontaneous fermentations during the production of the artisanal Brazilian cachaça. Can J Microbiol. 2002 May;48:5.

32. Volschenk H, van Vuuren HJJ, Viljoen-Bloom M. Malo-ethanolic fermentation in Saccharomyces and Schizosaccharomyces. Curr Genet. 2003 Sep;43:6. doi:10.1007/s00294-003-0411-6

33. Jacobs JZ, Ciccaglione KM, Tournier V, Zaratiegui M. Implementation of the CRISPR-Cas9 system in 
492

493

494

495

496

497

498

499

500

501

502

503

504

505

506

507

508

509

510

511

512

513

514

515

516

517

518

519

520

521

522

fission yeast. doi:10.1038/ncomms6344

34. Zhao Y, Boeke JD. Construction of Designer Selectable Marker Deletions with a CRISPR-Cas9 Toolbox in Schizosaccharomyces pombe and New Design of Common Entry Vectors. 2018; doi:10.1534/g3.117.300363

35. Zhang X-R, He J-B, Wang Y-Z, Du L-L. A Cloning-Free Method for CRISPR/Cas9-Mediated Genome Editing in Fission Yeast. 2018; doi:10.1534/g3.118.200164

36. Kurtzman CP. Biotechnological strains of Komagataella (Pichia) pastoris are Komagataella phaffii as determined from multigene sequence analysis. J Ind Microbiol Biotechnol. 2009 Nov;36:11. doi:10.1007/s10295-009-0638-4

37. Weninger A, Fischer JE, Raschmanová H, Kniely C, Vogl T, Glieder A. Expanding the CRISPR/Cas9 toolkit for Pichia pastoris with efficient donor integration and alternative resistance markers. J Cell Biochem. 2018;119:4. doi:10.1002/jcb.26474

38. Weninger A, Hatzl AM, Schmid C, Vogl T, Glieder A. Combinatorial optimization of CRISPR/Cas9 expression enables precision genome engineering in the methylotrophic yeast Pichia pastoris. J Biotechnol. 2016;235. doi:10.1016/j.jbiotec.2016.03.027

39. Gorter De Vries AR, De Groot PA, Van Den Broek M, Daran J-MG. CRISPR-Cas9 mediated gene deletions in lager yeast Saccharomyces pastorianus. Microb Cell Fact. 2017;16. doi:10.1186/s12934-0170835-1

40. Smith JD, Schlecht U, Xu W, Suresh S, Horecka J, Proctor MJ, et al. A method for high-throughput production of sequence-verified DNA libraries and strain collections. Mol Syst Biol. 2017;13. doi:10.15252/msb.20167233

41. Shi S, Liang Y, Zhang MM, Ang EL, Zhao H. A highly efficient single-step, markerless strategy for multi-copy chromosomal integration of large biochemical pathways in Saccharomyces cerevisiae. Metab Eng. 2016 Jan;33. doi:10.1016/J.YMBEN.2015.10.011

42. Jakočiūnas T, Jensen ED, Jensen MK, Keasling JD. Assembly and Multiplex Genome Integration of Metabolic Pathways in Yeast Using CasEMBLR. In Humana Press, New York, NY; 2018. p. 185-201. doi:10.1007/978-1-4939-7295-1_12

43. Lian J, HamediRad M, Hu S, Zhao H. Combinatorial metabolic engineering using an orthogonal trifunctional CRISPR system. Nat Commun. 2017 Dec;8:1. doi:10.1038/s41467-017-01695-x

44. Li Z-H, Liu M, Wang F-Q, Wei D-Z. Cpf1-assisted efficient genomic integration of in vivo assembled DNA parts in Saccharomyces cerevisiae. Biotechnol Lett. 2018 Aug;40:8. doi:10.1007/s10529-018-2574-8 
524 to inducing multiple chromosome splitting in Saccharomyces cerevisiae. Sci Rep. 2016 Sep;6:1. doi:10.1038/srep30278

46. Deaner M, Mejia J, Alper HS. Enabling Graded and Large-Scale Multiplex of Desired Genes Using a Dual-Mode dCas9 Activator in Saccharomyces cerevisiae. ACS Synth Biol. 2017 Oct;6:10. doi:10.1021/acssynbio.7b00163

47. Lee NCO, Larionov V, Kouprina N. Highly efficient CRISPR/Cas9-mediated TAR cloning of genes and chromosomal loci from complex genomes in yeast. Nucleic Acids Res. 2015 Apr;43:8. doi:10.1093/nar/gkv112

48. Ryan OW, Cate JHD. Multiplex Engineering of Industrial Yeast Genomes Using CRISPRm. In: Methods in enzymology. 2014. p. 473-89. doi:10.1016/B978-0-12-801185-0.00023-4

49. Schwartz C, Curtis N, Löbs A-K, Wheeldon I. Multiplexed CRISPR Activation of Cryptic Sugar Metabolism Enables Yarrowia Lipolytica Growth on Cellobiose. Biotechnol J. 2018 Sep;13:9. doi:10.1002/biot.201700584

50. Kang H-S, Charlop-Powers Z, Brady SF. Multiplexed CRISPR/Cas9- and TAR-Mediated Promoter Engineering of Natural Product Biosynthetic Gene Clusters in Yeast. ACS Synth Biol. 2016 Sep;5:9. doi:10.1021/acssynbio.6b00080

51. Deaner M, Alper HS. Systematic testing of enzyme perturbation sensitivities via graded dCas9 modulation in Saccharomyces cerevisiae. Metab Eng. 2017 Mar;40. doi:10.1016/j.ymben.2017.01.012

52. Jensen ED, Ferreira R, Jakočiūnas T, Arsovska D, Zhang J, Ding L, et al. Transcriptional reprogramming in yeast using dCas9 and combinatorial gRNA strategies. Microb Cell Fact. 2017 Dec;16:1. doi:10.1186/s12934-017-0664-2 